\title{
Digitalisierung und Logistik
}

\section{Ergebnisse aus dem BMBF-Verbundprojekt "Gesundheitsförderliche Arbeitsgestaltung für digitalisierte Dispositions- und Dokumentationsaufgaben in der Logistik" (Pro-DigiLog)}

\author{
Alexandra Schmitz, Fuyin Wei, Hans Uske und Bernd Noche
}

\subsection{Darstellung Vorgehen}

Das BMBF-geförderte Verbundprojekt „Gesundheitsförderliche Arbeitsgestaltung für digitalisierte Dispositions- und Dokumentationsaufgaben in der Logistik“ (Pro-DigiLog) betrachtete die Digitalisierung der Arbeit in der Logistikbranche und deren Auswirkungen auf die Beschäftigten und Unternehmen.

In diesem Beitrag sollen die Ergebnisse des Forschungsprojektes knapp zusammengefasst werden, indem die ingenieurwissenschaftlichen Entwicklungen sowie eine Diskussion im Projekt vorgestellt werden. Dabei wird zunächst die Frage aufgeworfen, was Digitalisierung in der Logistik bedeutet und welche Technik dahintersteht. Dabei wird aufgezeigt, wie sich Dokumentationsprozesse in der Logistik mit moderner Technik durch ein Verfahren, das im Projekt Pro-DigiLog entwickelt wurde, kostengünstig digitalisieren lassen. Die zweite Hälfte des Beitrags zieht ein Resümee mithilfe von sieben aufgestellten Thesen zur Digitalisierung in der Logistik und den Möglichkeiten gesundheitsförderlicher Arbeitsgestaltung.

\footnotetext{
A. Schmitz $(\bowtie) \cdot H$. Uske

Rhein-Ruhr-Institut für Sozialforschung und Politikberatung (RISP),

Duisburg, Deutschland

F. Wei $\cdot$ B. Noche

Universität Duisburg-Essen, Transportsysteme und -logistik (TuL),

Duisburg, Deutschland
} 


\subsection{Präsentation Forschungsergebnisse}

Im Projekt Pro-DigiLog wurden zwei technische Richtungen zur Optimierung logistischer Prozesse insbesondere für kleine und mittlere Unternehmen untersucht: Zum einen die digitalen Dokumentationsprozesse in Unternehmen und zum anderen Sensortechniken zur Steuerung von Logistikprozessen. In diesem Beitrag wird näher auf die digitalen Dokumentationsprozesse eingegangen. ${ }^{1}$

Um die Bedeutung der Digitalisierung in der Logistik zu erfassen, muss zunächst näher auf den Status quo der Digitalisierung in der Logistik und auf die Logistikbranche im Allgemeinen eingegangen werden. Der Logistiksektor in Deutschland ist geprägt durch den Mittelstand [8]. Annähernd $99 \%$ der Unternehmen im Logistikmarkt sind kleine oder mittlere Unternehmen (KMU) [7]. Die Strukturen dieses Geschäfts sind gezeichnet von geringen Margen, resultierend aus geringen Eintrittsbarrieren in den Markt und dem enormen Wettbewerbsdruck [9]. Ein weiteres Merkmal ist der hohe Anteil der Personalkosten. Sie entstehen auf Basis des hohen Koordinationsaufwandes, der vornehmlich auf Papier abgewickelt wird. Der Trend der Digitalisierung fordert erhöhte Flexibilität und Transparenz in der gesamten Wertschöpfungskette. Wer diese und spezielle Kundenanforderungen nicht erfüllen kann, hat angesichts der großen Konkurrenz auf dem Markt Existenzproblematiken [10, 14].

Die Nutzung neuer Technologien ermöglicht Optimierung von Logistikketten. Daher sind deutschlandweit sowohl Start-Ups als auch etablierte Unternehmen bemüht, die Logistik mithilfe von Technologien zu verändern. Dafür sind Ansätze entwickelt worden, wie z. B. Plattformen zum Teilen von Lagerraum, Auslieferungsroboter für die Paketzustellung oder Module zur Containerüberwachung. Die ersten Angebote sind am Markt angekommen und viele weitere befinden sich in der Entwicklung [11]. Zukünftig und langfristig gesehen sollen durch die Integration neuer Technologien Maschinen, Menschen, Produkte und sogar Produktions- und Lagersysteme in die Lage versetzt werden, kontinuierlich Informationen untereinander auszutauschen.

\subsubsection{Digitalisierung und digitale Dokumentationsprozesse}

Die unternehmensseitigen Ziele entsprechender Anwendungen sind vielseitig: neben der Verbesserung von Prozessen und Kapazitätsauslastung, spielt auch die raschere Umsetzung individueller Kundenwünsche im Wertschöpfungsprozess eine große Rolle. Die Verflechtung der jeweiligen physischen Gegebenheiten mit einer virtuellen Umgebung zeigt sich oftmals jedoch nur dann aussichtsreich, wenn das betreffende Unternehmen über stabile und ausreichend dokumentierte prozessuale Infrastrukturen

\footnotetext{
${ }^{1}$ Für die Ergebnisse der untersuchten Sensortechnik verweisen wir auf unseren Sammelband „Logistik und Digitalisierung“ [5] veröffentlicht im März 2020.
} 


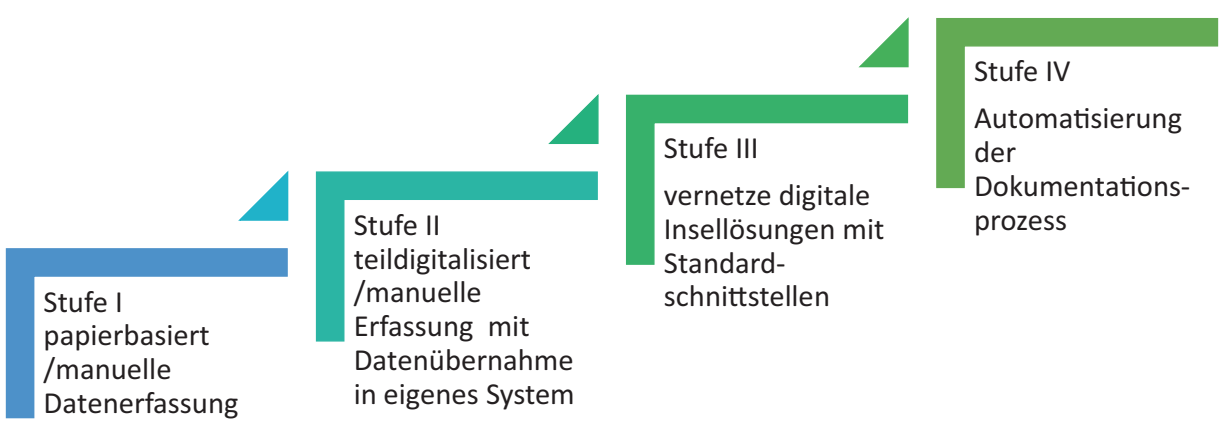

Abb. 30.1 Reifegradmodell des digitalen Dokumentationsprozesses

verfügt. Ziel der Ausarbeitungen im Forschungsprojekt Pro-DigiLog ist es, den Wandel im Zuge der Digitalisierung der logistischen Arbeitswelt von seinen Anfängen bis hin zu heutigen technologischen Möglichkeiten zu beleuchten. Darüber hinaus sollen auch potenzielle Anwenderinnen und Anwender von den beschriebenen Technologien profitieren, indem sie mit diesen konfrontiert und für deren Umgang im Alltag sensibilisiert werden. Auf dieser Grundlage wird anhand verschiedener Stufen der Dokumentationsprozesse (Reifegradmodell) schrittweise dargestellt, wie digitale Infrastrukturen Einzug in eine logistische Prozesslandschaft erhalten können.

Grundlage hierfür bildet eine vollständig papierbasierte Abwicklung (Stufe I) der beschriebenen Abläufe. Diese wird im Rahmen der Forschung um verschiedene technologische Komponenten ergänzt. Darunter fallen die Verwendung rudimentärer Informationstechnologie zur Stabilisierung der Abläufe, beispielsweise teildigitalisiert, manuelle Erfassung mit Datenübernahme in das eigene E-Dokumentenmanagement System (Stufe II) bis hin zum Einsatz einer vernetzten internen digitalen Lösung mit Standardschnittstellen, wie z. B. ein geeignetes Warehouse Management System (Stufe III), welches der Anwenderin/dem Anwender verschiedene Möglichkeiten zur Geschäftsprozesssteuerung und -optimierung bietet. Darauf aufbauend werden Potenziale und Herausforderungen einer Einführung von OCR-Technologie (Texterkennung oder auch optische Zeichenerkennung; Abkürzung von Englisch „Optical Character Recognition“) für die Automatisierung der Dokumentation (Stufe IV) veranschaulicht (Abb. 30.1).

Im Rahmen des Forschungsprojekts Pro-DigiLog haben wir zusammen mit unserem Anwendungspartner in einer Pilotinsel versucht, die Messdaten aus verschiedenen Maschinen automatisch zu dokumentieren.

Wegen des heterogenen Datenstandards und vielfältigen Maschinenschnittstellen von unterschiedlichen Maschinenherstellern, werden heutzutage noch in vielen Unternehmen Messdaten von Mitarbeiterinnen und Mitarbeitern abgelesen und schriftlich auf ein papierbasiertes Formular übertragen. Diese Zettel werden dann in Ordnern archiviert. Das Verfahren ist arbeitsintensiv, fehleranfällig und die Daten können schlecht ausgewertet werden. Neue oder modernere Maschinen können heutzutage schon die 
Daten direkt an eine Datenbank senden, allerdings sind diese Maschinen relativ teuer. Alte Maschinen können gar nicht oder nur recht aufwendig nachgerüstet werden. Bei neuen Maschinen ist eine Integration in die aktuellen Prozessabläufe mit anderen schon existierten Maschinen oft nicht vorgesehen.

Insbesondere die kleinen und mittleren Unternehmen brauchen ein neues digitales Dokumentationsverfahren. Es muss einfach, preisgünstig und sicher sein. Aus diesem Grund, haben wir die OCR-Technologie ausgewählt, um die Dokumentationsprozesse bei der heterogenen Maschinenumwelt zu automatisieren und $\mathrm{zu}$ verbessern. Analoge Messdaten aus unterschiedlichen Maschinen sollen automatisch in ein digitales Formular übertragen werden. Es sollte besser und fehlerfreier ausgewertet werden. Im Projekt ProDigiLog wurde ein automatisches Verfahren der digitalen Dokumentation entwickelt. Für das neue Verfahren sind fünf Schritte nötig:

- Ein Gerät mit einer Kamera, das Aufnahmen mit mehr als 5 Megapixel machen kann.

- Ein/-e Mitarbeiter/-in, der/die die veränderten Arbeitsschritte durchführt.

- Eine Texterkennungssoftware

- Eine Datenbank

- Die Speicherung der Daten mit verschiedenen digitalen Ausgabenformaten.

Im Folgenden werden die Abläufe anhand eines Beispiels dargestellt. Im ersten Schritt geht der Mitarbeiter zu einem Messgerät. Dort fotografiert er die Daten mit einem Smartphone. Damit man weiß, wer die Aufnahme gemacht hat, legt er seinen Ausweis dazu. Dann schickt der Mitarbeiter das Foto per WLAN an einen Computer. Dort landet das Foto in einem digitalen Ordner. Durch den Einsatz einer Texterkennungssoftware werden die Daten auf dem Foto digitalisiert. Mithilfe dieser Software ist es uns gelungen, Daten aus verschiedenen Messgeräten erfolgreich zu digitalisieren. Die Daten können nun in einer Datenbank sicher gespeichert werden. Auch können sie jetzt ausgewertet und weiterverarbeitet werden. Die Ergebnisse sehen so aus: die Dokumente, die bisher per Hand ausgefüllt wurde, können nun automatisch und sauber gelesen und als pdf-Datei gespeichert werden. Die Daten können nun besser archiviert und auch besser ausgewertet werden (Abb. 30.2).

Dieses Verfahren ist relativ kostengünstig und damit auch geeignet für kleine und mittlere Unternehmen. Im Vergleich zu den alten analogen Prozessen ist es sicherer und wenig fehleranfällig. Anhand dieser prototyphaften Entwicklung, konnte gezeigt werden, dass eine wirtschaftliche Nutzung möglich ist. Um die Produkt-Serienreife zu erreichen, muss die Lösung allerdings noch mehrfach in der Praxis getestet werden.

Zwar bauen die einzelnen Stufen der Dokumentationsprozesse aufeinander auf, jedoch sind die einzelnen Betrachtungen darauf ausgelegt, dass sie auch isoliert genutzt werden können. Neben einer grundlegenden Einführung in die betrachtete Thematik werden demnach stets mögliche Anwendungsfälle und Schritte zur Umsetzung der jeweiligen technologischen Komponenten diskutiert. Das abschließende Fazit dieser Arbeit soll daher bewusst kein Resümee aus der einzelnen Stufe ziehen, sondern viel- 


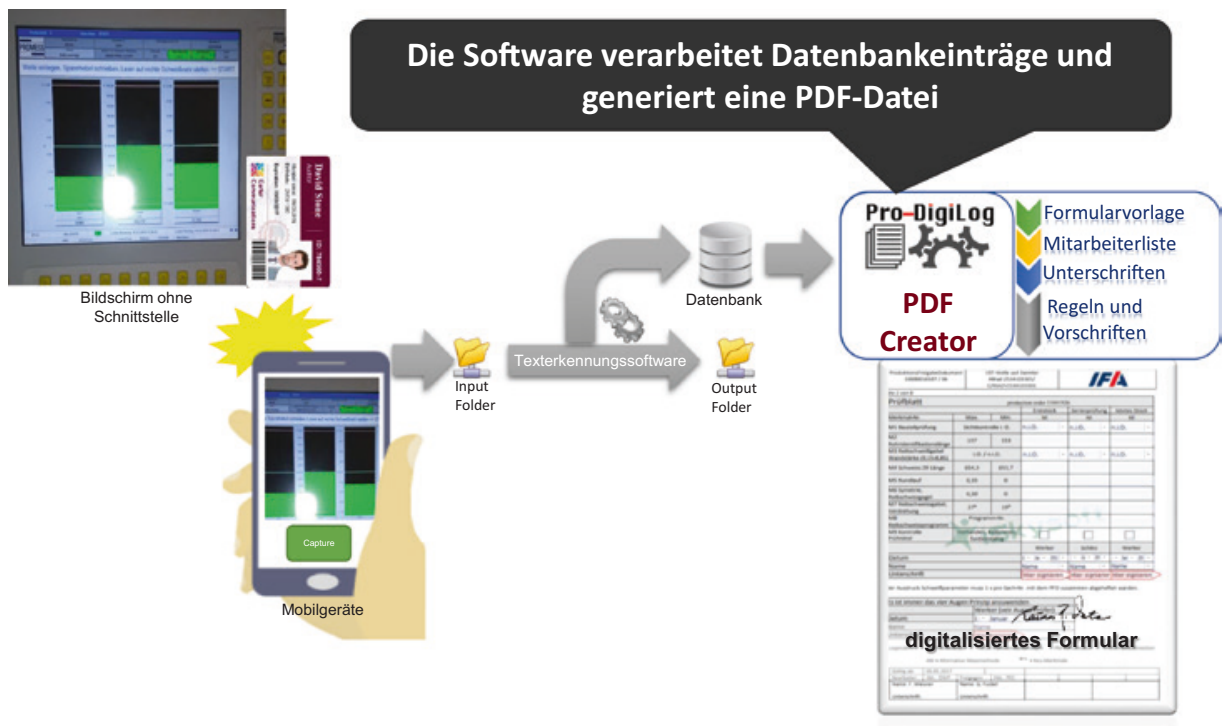

Abb. 30.2 Automatisierung der Übertragung von analogen Daten zum digitalisierten Formular

mehr einen Ausblick auf weiterführende technologische Möglichkeiten der logistischen Prozessabwicklung geben. Dadurch bieten sich erste Denkanstöße zur Weiterentwicklung der hier beschriebenen Digitalisierungsmaßnahmen.

\subsubsection{Thesen zur Digitalisierung in der Logistik und den Möglichkeiten gesundheitsförderlicher Arbeitsgestaltung}

Über die technische Seite des Projektes Pro-DigiLog hinaus, wird anhand von sieben aufgestellten Thesen die Diskussion um die Digitalisierung in der Logistik abgebildet, die als Resultat des Projekts festgehalten werden kann.

\section{Szenarien zur Digitalisierung - Inwieweit prägt die Digitalisierung die Zukunft der Arbeit?}

In der Literatur werden aktuell im Wesentlichen vier Szenarien zur Zukunft der Arbeit diskutiert. Im Negativszenario werden viele Tätigkeiten durch digitale Technologien ersetzt. Im Positivszenario werden sie $\mathrm{zu}$ höherwertigen Tätigkeiten. Im Polarisierungsszenario entsteht eine Schere zwischen komplexer und einfacher Arbeit, wobei die mittlere Qualifikationsgruppe (Facharbeit) an Bedeutung verliert. Im Entgrenzungszenario schließlich werden traditionelle Arbeiten durch neue Arbeitsformen ersetzt [2]. Umstritten ist auch das Verhältnis von Digitalisierung und Arbeit. Während in vielen Publikationen der Digitalisierung eine eindeutige Prägekraft auf Arbeit zugeschrieben wird, betonen andere Autoren die Bedeutung von Leitbildern, 
Organisationskonzepte und Aushandlungsprozesse bei der Analyse des Zusammenhangs von Technik und Arbeit [1, 4].

Die Analysen und Erfahrung aus dem Projekt ProDigiLog stützen diese These. Digitalisierung verändert die Arbeitsprozesse in der Logistik, determiniert aber nicht die Arbeitsbedingungen und die Möglichkeiten der Arbeitsgestaltung.

\section{Digitalisierung in der Logistik hat eine spektakuläre und eine weniger spektakuläre Seite.}

Während in der öffentlichen Diskussion von künftig selbstfahrenden LKWs die Rede ist, sucht die Branche händeringend nach LKW-Fahrern. Während schon vor Jahren vorhergesagt wurde, dass fahrerlose Gabelstapler die Zukunft in den Lägern prägen wird, werden weiterhin Gabelstaplerfahrer ausgebildet und gesucht. Und während in Zeitungsartikeln autonom fahrende Binnenschiffe künftig den Rhein-Herne-Kanal befahren, verweisen Praktiker auf die dabei nicht berücksichtigten Schwierigkeiten [6].

Es gibt zwei Formen der Digitalisierung. Die spektakuläre Seite, die die öffentliche Wahrnehmung prägt, findet vor allem in Großbetrieben und in darauf zugeschnittenen Forschungen statt. Was dort an veränderten Arbeitsformen entwickelt oder prognostiziert wird, ist nicht die Zukunft der Arbeit, die mittelfristig gesehen in den meisten Bereichen der KMU-dominierten Logistik stattfinden wird. Das heißt nicht, dass in diesen Betrieben analog weitergearbeitet wird. Digitalisierung findet hier aber in einer anderen Form, auf einem anderen Level, wenig spektakulär und öffentlich selten sichtbar statt. An solchen Formen der Digitalisierung arbeitet das Projekt Pro-DigiLog.

\section{Digitalisierung bei Logistik KMU und Großunternehmen: Die Unterschiede vergrößern sich. Das hat Folgen.}

Wie sinnvoll es ist, unspektakuläre Formen der Digitalisierung in der Logistik voranzubringen, zeigt eine Studie, die im Projekt Pro-DigiLog erstellte wurde und die auf einer Online-Befragung von Logistikunternehmen sowie 40 Expertinnen- und Experteninterviews beruht [6]. Die Studie kommt zu dem Ergebnis, dass der Grad der Digitalisierung in den kleinen und mittleren Logistikunternehmen noch nicht so ausgebaut ist wie in den größeren. Der überwiegende Teil der befragten Unternehmen sieht sich selbst als wenig oder eher wenig digitalisiert. Dies deckt sich mit Ergebnissen einer größeren OnlineBefragung, die das Bundesinstitut für Berufsbildung (BiBB) 2018 bei Logistikunternehmen durchgeführt hat. Zentrales Ergebnis war, dass fortschrittliche Unternehmen heute bereits „4.0“-Tätigkeiten und -Kompetenzen definieren, bei kleinen und mittleren Betrieben sei die Digitalisierung aber noch nicht angekommen.

Zur Zukunft des Berufs Fachkraft für Lagerlogistik gefragt, entwickelt das BiBB zum Beispiel folgendes Szenario: „Eine Fachkraft für Lagerlogistik ,4.0“ beispielsweise ist nicht mehr der ,Kistenschubser', sondern befasst sich mit Prozesssteuerung und -optimierung, arbeitet im Leitstand und ist verantwortlich für die Datenpflege und die Qualitätssicherung“ [3]. Wenn aber die Fachkraft für Lagerlogistik in Kleinbetrieben 
ausgebildet wird, wird sie mit dieser Technik nicht oder nur selten in Berührung kommen.

Und dies gilt nicht nur für die Ausbildung und die damit zusammenhängenden Probleme. Die Lücke zwischen der High-Tech-Logistik in Großbetrieben und analogen oder wenig digitalisierten Techniken in KMU wird größer und damit für letztere zum Problem. Denn einerseits sind sie vernetzt und eingebunden in logistische Ketten mit digital agierenden Akteuren. Andererseits stellt Digitalisierung erhebliche unausgeschöpfte Produktivitätsreserven dar. Es besteht also durchaus ein Handlungsdruck auch für KMU.

4. Durch die Digitalisierung gibt es zunehmend technische Möglichkeiten für KMU, die für gesundheitsförderliche Arbeitsgestaltung einsetzbar sind. Dies bedeutet aber nicht, dass sie auch zur Anwendung kommen.

Im Projekt Pro-DigiLog konnten vor dem Hintergrund verbesserter und preisgünstiger werdender Sensortechnik Verfahren entwickelt werden, die dazu beitragen können, Arbeit effektiver aber auch gesundheitsförderlicher zu gestalten. Die Überlegungen, die beim Projektpartner IFA dazu durchgeführt wurden, haben gezeigt, dass diese Technik Hinweise auf Gesundheitsgefahren liefern, die dann behoben werden könnten.

Besondere Einsatzmöglichkeiten ergeben sich beispielsweise durch die Nutzung von Wearables. Dabei werden die Mitarbeiter mit Sensoren ausgestattet (wie z. B. Schrittzähler, Pulsmesser, Smart Watches (und Brillen) aber auch RFID Chips zur Zugangskontrolle).

Es gibt erhebliche Widerstände beim Einsatz dieser Techniken bei einem Teil der Belegschaft, da bei einzelnen Mitarbeitern ein Gefühl der permanenten Überwachung entsteht. Der Nutzen des Einsatzes dieser Techniken reduziert sich aber auch dadurch, dass die Mitarbeiter gezwungen sind die Systeme ständig zu tragen bzw. anzulegen. Dies kann aber nicht immer gewährleistet werden.

\section{Eine automatische und kostengünstige Verwandlung umfangreicher, verschieden erhobener analoger Daten in digitalisierter Form ist möglich.}

Einer der zentralen Entwicklungen im Projekt Pro-DigiLog ist die reibungslose Umwandlung von analogen Daten in weiter verarbeitbare digitale Daten. Dazu sind Versuchsanordnungen beim Projektpartner IFA durchgeführt worden, die dann am Lehrstuhl Transportsysteme und -logistik (TUL) der Universität Duisburg-Essen weiterbearbeitet wurden.

In vielen Unternehmen werden heute noch Messdaten von Mitarbeitern abgelesen und schriftlich auf ein Formular übertragen. Die Zettel werden dann in Ordnern archiviert. Eine Alternative wäre, wenn die Maschinen die Daten direkt an eine Datenbank senden. Das ist praktisch, aber auch sehr teuer und daher für viele Unternehmen nicht machbar. Kleine und mittlere Unternehmen brauchen ein Dokumentationsverfahren, das einfach, preisgünstig und sicher ist. Im Projekt Pro-DigiLog wurde ein solches Verfahren entwickelt. In einem Film ist dieses Verfahren dokumentiert. Allerdings ist dies zunächst ein 
Prototyp. In der Verwertung wird sich zeigen, ob dieses Verfahren auch allgemein nutzbar gemacht werden kann. Dann erst lässt sich verlässlich sagen, welche Arbeitsprozesse sich dabei verändern und welche Auswirkungen das für die Beschäftigten hat.

\section{These zur Digitalisierung bei Logistikdienstleistern.}

Die Anwendungen der Digitalisierung konzentrieren sich auf die operative Durchführung der Arbeiten. Dabei kann man grundsätzlich unterscheiden zwischen: Arbeitsumgebung, Arbeitsprozesse, Infrastruktur, Qualitätsinformationen und Hilfsprozesse.

Bei der Arbeitsumgebung können die Bedingungen unter denen die Arbeit verrichtet wird überwacht werden. Dazu gehören beispielsweise die Raumtemperatur und die Luftfeuchtigkeit sowie die Zusammensetzung der Luft oder auch die Lichtverhältnisse. Es lassen sich damit Arbeitsbedingungen identifizieren, die zu erhöhten Krankheitsständen und zum Unwohlsein führen.

Auf der Prozessebene der können beispielsweise Informationen zur Arbeitsdichte, der Arbeitshaltung, der Handhabungsgewichte, Wartezeiten, der zurückgelegten Wege ermittelt werden. Darauf aufbauend können Strategien zur Vermeidung von temporärer Überlast und zur Balancierung der Arbeiten im Team entwickelt werden.

Auf der Ebene der Infrastruktur können Daten erhoben werden, die den Arbeitsfluss charakterisieren. So können beispielsweise Pufferbelegungen, Hindernisse auf den Wegen, Anordnungen von Bereitstellungen, Bestände von Hilfsstoffen und Ausfallzeiten der Energieversorgung dokumentiert werden. Diese Informationen können genutzt werden um Gefahren, Wegezeiten, Suchaufwände und weitere überflüssige Aktionen zu vermeiden, die sich auf Ursachen der Infrastruktur zurückführen lassen.

Auf der Qualitätsebene können vielfältige produkt- und prozessbezogene Informationen gewonnen werden die beispielsweise den Zustand der Maschinen oder die Qualität der Produkte definieren gewonnen werden. Damit können Nacharbeiten und Rüstaufwände beeinflusst werden. Dies kann die Motivation der Mitarbeiter erhöhen und die Arbeitsatmosphäre verbessern.

$\mathrm{Zu}$ den Hilfsprozessen gehören beispielsweise die Instandhaltung, Reinigung von Behältern, Zusammen- und Bereitstellung von Materialien (Kits oder Verpackungsmaterial). Mithilfe der Digitalisierung lassen sich Engpässe frühzeitig ermitteln und ein Verbrauchsmonitoring installieren. Dadurch werden Eilaufträge und Notmaßnahmen sowie Produktionsunterbrechungen vermieden. Es entstehen seltener Stresssituationen und auch Zusatzarbeiten mit Überstunden können reduziert werden.

\section{Digitalisierung kann Arbeit erleichtern oder erschweren. Sie kann gesundheits- förderlich gestaltet werden oder die Gesundheit gefährden.}

Im Projekt Pro-DigiLog wurde ein Ausschnitt der Logistik untersucht. Es wurden mithilfe digitaler Techniken Entwicklungsmöglichkeiten aufgezeigt, die die Arbeit effektiver und gesundheitsförderlicher gestalten können. Dies ist nicht selbstverständlich.

Analysen der Gewerkschaft deuten darauf hin, dass die Mehrheit der Beschäftigten in allen Branchen eine Belastungszunahme durch den Einsatz digitaler Arbeitsmittel 
wahrnimmt, nur eine Minderheit berichtet über Erleichterungen. Dies gilt auch und gerade für die Logistikbranche [2]. Auf die Frage inwieweit sich die Arbeitsbelastung durch die Digitalisierung verändert habe, antworteten $41 \%$ der Befragten im Bereich Verkehr und Lagerei, sie sei eher größer geworden. Nur $8 \%$ waren der Ansicht, sie sei geringer geworden (ebenda S. 76 [2]).

Wie kompliziert der Zusammenhang zwischen Digitalisierung und Arbeitsgestaltung sein kann zeigt das Beispiel der Paketdienstfahrer, deren Arbeitsbedingungen aktuell in der Öffentlichkeit als besonders belastend beschrieben werden [15]. Verantwortlich sei vor allem die Praxis, Sub-Unternehmen für die Zustellung der Pakete einzusetzen.

Am Beispiel der KEP-Branche (Kurier-, Express-, Paketdienste) zeigt sich in der Tat, dass Digitalisierung gleichzeitig Arbeitsprozesse erleichtert und erschwert. In welcher Form und mit welcher Intensität dies geschieht, ist nicht technisch determiniert, sondern hängt von der Struktur der Arbeitsbeziehungen, Machtfragen, Verhandlungsgeschick und staatlichen Regulierungen ab. Dagmar Wäscher, Kooperationspartnerin im Projekt Pro-DigiLog, langjährige Subunternehmerin und Vorsitzende des Bundesverbandes der Transportunternehmen (BVT) hat diese Zusammenhänge für den Abschlussband des Projektes beschrieben:

Früher haben Subunternehmer und deren Fahrer die Paket-Nummer, den Empfänger und die Adresse handschriftlich auf Rolllisten erfasst. Aufgrund der schnell steigenden Paketmengen war dies nach ein paar Jahren zeitlich nicht mehr möglich und es wurden Handscanner eingeführt. Hierdurch wurde die Paketerfassung wesentlich schneller und die Mehrmengen können bis heute in einer angemessenen Zeit bewältigt werden. Diese Einführung war für Subunternehmer und Fahrer zunächst eine enorme Erleichterung. Heute sind die Fahrer durch die Scanner mit GPS-Anbindung ständig überwacht und haben fast keinen Einfluss mehr auf ihre Routenplanung, weil sie eine bestimmte Route einhalten müssen, damit der Kunde weiß, wann sein Paket kommt. Der Zusteller hat viel an Eigenverantwortung und Freiheit verloren. Konnte er früher seine Route frei planen, muss er heute die vorgeschriebene Route einhalten. Das geht soweit, dass, wenn er aus dem Zeitplan kommt, der Scanner nicht mehr arbeitet und durch einen Mitarbeiter in der Niederlassung wieder freigeschaltet werden muss. Zudem können ihm jederzeit auf den Scanner zusätzliche Aufträge übermittelt werden, von denen der Subunternehmer (Arbeitgeber des Fahrers) nichts weiß und die Arbeitszeitplanung somit zunichtemacht. Damit riskiert der Subunternehmer gesetzliche Vorschriften nicht mehr einhalten zu können, für die er dann haftet [12].

\subsection{Fazit}

Im Antrag zum Projekt Pro-DigiLog haben wir zwei Szenarien beschrieben, mögliche Entwicklungspfade der Digitalisierung. Im ersten Szenario wird Digitalisierung allein unter dem Gesichtspunkt der betrieblichen Leistungspolitik gestaltet. Dies bedeutet dann: Nochmalige Intensivierung der Arbeit, stärkere Überwachung, Vervoll- 
kommnung der tayloristisch geprägten Arbeitsteilung. Für den Arbeits- und Gesundheitsschutz bedeutet das, dass Beschäftigte unabhängig von ihrem Alter und Geschlecht mit zunehmender Belastung konfrontiert werden, bei gleichzeitiger Unmöglichkeit Einfluss nehmen zu können, weder bei der Gestaltung der Technik noch bei der daran gekoppelten Form der Arbeitsorganisation.

Bei Szenario 2 wird bereits bei der Technikentwicklung mitüberlegt, wie die Digitalisierung der Produktion Arbeitsabläufe förderlicher für den Arbeits- und Gesundheitsschutz gestalten kann - ohne die Gesamtleistung zu vermindern! Der Nutzen für das Unternehmen: Weniger Fehlzeiten, niedrigere Krankenstände, motiviertere Mitarbeiterinnen und Mitarbeiter, bessere Rekrutierungschancen auch im Demografischen Wandel mit dem Image „Gesunder Betrieb“, geringere Fehlerraten und Bearbeitungszeiten, weniger Korrekturen und Störungen. Das Verbundprojekt will zeigen, dass das Szenario 2 für kleine und mittlere Unternehmen der Logistikbranche machbar, realistisch, nachhaltig und zukunftsweisend ist.

An dieser Aussage halten wir fest auch wenn die Praxis in vielen Branchensegmenten eher zu Szenario 1 tendiert:

- Die von der Universität Duisburg-Essen, Lehrstuhl Transportsysteme und -logistik (TUL) entwickelten Techniken im Projekt Pro-DigiLog erlauben es KMU, Digitalisierung kostengünstig und gesundheitsförderlich zu gestalten.

- Das Teilprojekt der timestudy GmbH hat gezeigt, dass ergonomische Studien für die Unternehmen dazu einen wertvollen Beitrag leisten können.

- Gespräche mit kleinen und mittleren Unternehmen in der Logistik, die vor allem vom Projektpartner VSL (Verband Spedition und Logistik NRW), aber auch von anderen Teilprojekten durchgeführt wurden, haben gezeigt, dass bei vielen Unternehmen durchaus ein Interesse an entsprechenden Lösungskonzepten existiert.

- Dies belegt auch die Studie „Arbeit und Logistik 2025“, die federführend vom Projektpartner RISP durchgeführt wurde. Als Fazit der dort geführten Interviews folgert die Studie: „Die Logistikarbeit der Zukunft digital und gesund zu gestalten liegt grundsätzlich im Interesse der Unternehmen, auch um als Arbeitgeber attraktiv für zukünftige Mitarbeiterinnen und Mitarbeiter zu sein“.

\section{Projektpartner und Aufgaben}

- Universität Duisburg-Essen (UDE) Entwicklung von Standards für Dispositions- und Dokumentationsaufgaben

- Rhein-Ruhr-Institut für Sozialforschung und Politikberatung (RISP) Analyse künftiger Arbeitsgestaltung

- Verband Spedition und Logistik Nordrhein-Westfalen e. V. (VSL) Verbreitungsmöglichkeiten in der Distributionslogistik 


\section{- Time Study GmbH}

Konzepte für Beraterfirmen in der digitalisierten Logistik

- IFA GmbH \& Co.KG

Pilotunternehmen

\section{Literatur}

1. Baethge V, Kuhlmann M, Tullius K (2018) Technik und Arbeit in der Arbeitssoziologie Konzepte für die Analyse des Zusammenhangs von Digitalisierung und Arbeit. Arbeits- und Industriesoziologische Studien 11(2):91-106

2. DGB-Index Gute Arbeit (2017) Verbreitung, Folgen und Gestaltungsaspekte der Digitalisierung in der Arbeitswelt. Auswertungsbericht auf Basis des DGB-Index Gute Arbeit 2016

3. Kaufmann A, Kock A (2018) „Fördernde und hemmende Faktoren für die Gestaltung der Berufsbildung" - Fachtagung Fachkräftequalifikationen und Kompetenzen für die digitalisierte Arbeit von morgen. Ergebnisse und erste Handlungsempfehlungen

4. Kuhlmann M, Schumann M (2015) Digitalisierung fordert Demokratisierung der Arbeitswelt heraus. In: Hoffmann R, Bogedan C (Hrsg) Arbeit der Zukunft. Möglichkeiten nutzen - Grenzen setzen. Campus, Frankfurt, S 122-140

5. Schmitz A, Uske H, Noche B, Wei F (2020) Logistik und Digitalisierung. Ergebnis aus dem BMBF-Verbundprojekt „Gesundheitsförderliche Arbeitsgestaltung für digitalisierte Dispositions- und Dokumentationsaufgaben in der Logistik“ (Pro-DigiLog). Duisburg 2020

6. Schmitz A (2018) Arbeit und Logistik 2025, Eine Studie im Rahmen des Verbundprojektes Pro-DigiLog. https://www.risp-duisburg.de/media/studie_arbeit_und_logistik_2025.pdf. Zugegriffen: 03. März 2020

7. Baumgarten H (Hrsg) (2008) Das Beste der Logistik- Innovation, Strategien, Umsetzung. Springer, Berlin

8. Deutscher Speditions- und Logistikverband e.V. (DSLV) (2015) Zahlen Daten Fakten aus Spedition und Logistik. DSLV e.V., Berlin

9. Gronemeier T, Mutzke H (2014) Transport/Logistik- Branchenbericht- corporate sector report. Commerzbank AG, Frankfurt a. M.

10. Gundelfinger C, Naumann V, Pflaum A, Schwemmer M (2017) Transportlogistik 4.0. Erlangen: Fraunhofer- Institut für integrierte Schaltungen (IIS)

11. Maluck J, Nowak G, Pasemann J, Stürmer C (2016) The era of digitized truckingTransforming the logistics value chain. PricewaterhouseCoopers $\mathrm{GmbH}$, München

12. Wäscher D (2020) Die Entwicklung der Kurier-, Express- und Paketdienste (KEP) und die Rolle der Digitalisierung. In: Schmitz A, Uske H, Noche B, Wei F (Hrsg) Logistik und Digitalisierung. Duisburg 2020

13. Welzel P, Lühring M (2019) Die SUB SUB SUB-Masche. In: publik (2/2019) S 1

14. Wohlers E (2015) HWWI Policy Paper Nr. 92 - Logistik- ein wichtiger Wirtschaftsbereich in Deutschland. Hamburgisches Welt Wirtschaftsinstitut GmbH (HWWI), Hamburg 
Open Access Dieses Kapitel wird unter der Creative Commons Namensnennung 4.0 International Lizenz (http://creativecommons.org/licenses/by/4.0/deed.de) veröffentlicht, welche die Nutzung, Vervielfältigung, Bearbeitung, Verbreitung und Wiedergabe in jeglichem Medium und Format erlaubt, sofern Sie den/die ursprünglichen Autor(en) und die Quelle ordnungsgemäß nennen, einen Link zur Creative Commons Lizenz beifügen und angeben, ob Änderungen vorgenommen wurden.

Die in diesem Kapitel enthaltenen Bilder und sonstiges Drittmaterial unterliegen ebenfalls der genannten Creative Commons Lizenz, sofern sich aus der Abbildungslegende nichts anderes ergibt. Sofern das betreffende Material nicht unter der genannten Creative Commons Lizenz steht und die betreffende Handlung nicht nach gesetzlichen Vorschriften erlaubt ist, ist für die oben aufgeführten Weiterverwendungen des Materials die Einwilligung des jeweiligen Rechteinhabers einzuholen.

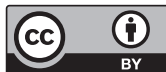

